\title{
SOME UNUSUAL EPICOMPLETE ARCHIMEDEAN LATTICE-ORDERED GROUPS
}

\author{
ANTHONY W. HAGER \\ (Communicated by Ken Ono)
}

\begin{abstract}
An Archimedean l-group is epicomplete if it is divisible and $\sigma$ complete, both laterally and conditionally. Under various circumstances it has been shown that epicompleteness implies the existence of a compatible reduced $f$-ring multiplication; the question has arisen whether or not this is always true. We show that a set-theoretic condition weaker than the continuum hypothesis implies "not", and conjecture the converse. The examples also fail decent representation and existence of some other compatible operations.
\end{abstract}

\section{INTRODUCTION}

Arch is the category of Archimedean l-groups, with l-group homomorphisms, fr $\mathbf{A}$ is the category of $f$-rings which are reduced (semi-prime) and Archimedean, with $l$-ring homomorphisms, and frA $\stackrel{F}{\rightarrow}$ Arch is the forgetful functor. " $G \in$ $F(\boldsymbol{f r} \mathbf{A})$ " means $G \in \operatorname{Arch}$ and for some multiplication * compatible with the $l$-group structure of $G,(G, *) \in$ fr $\mathbf{A}$.

In a general category, an object $A$ is called epicomplete (EC) if $\varphi: A \rightarrow \bullet$ monic and epic implies $\varphi$ is an isomorphism.

In Arch, monics are one-to-one, and BH90a shows: $G$ is EC if and only if $G$ is divisible, laterally and conditionally $\sigma$-complete; EC is monoreflective in Arch, and thus is the least monoreflective subcategory. (See HS07 regarding "monoreflective".)

Question 1.1 ([Hol03] and [BHJK09], p. 166). $G \mathrm{EC} \Rightarrow G \in F(\mathbf{f r A})$ ?

This has arisen because the implication does hold under some extra assumptions (described in 1.6 below). We shall show that the following set-theoretic hypothesis implies "No" (which was conjectured in [BHJK09]).

Hypothesis 1.2. For a set $S$ of cardinal $|S|=\aleph_{1}$, there is a family $\mathcal{W}$ of subsets of $S$ with the properties

(1) $\forall A \in \mathcal{W},|A|=\aleph_{1}$,

(2) $\forall A \neq B$ in $\mathcal{W},|A \cap B| \leq \aleph_{0}$, and

(3) $|\mathcal{W}|=2^{\aleph_{1}}$

Received by the editors November 19, 2013.

2010 Mathematics Subject Classification. Primary 06E20; Secondary 03E50, 06B15, 06F25, 08A99, 18A20, 18A40, 46E25, 54C30, 54G05.

Key words and phrases. Lattice-ordered group, $f$-ring, epicomplete, reflection, $\sigma$-complete, truncation, essential completion, continuum hypothesis, basically disconnected space. 
Baumgartner has shown (see Jec03], pp. 579, 582): In ZFC,

$$
2^{\aleph_{0}}=\aleph_{1} \Rightarrow\left(2^{\aleph_{0}}<2^{\aleph_{1}} \text { and } 2^{\aleph_{0}}<\aleph_{\omega_{1}}\right) \Rightarrow 1.2 \Rightarrow 2^{\aleph_{0}}<2^{\aleph_{1}} \text {. }
$$

Thus Hypothesis 1.2 is weaker than the continuum hypothesis (and consistent with ZFC). In the following, $|S|=\aleph_{1}$ and $\mathbb{R}^{S}$ (all functions $f: S \rightarrow \mathbb{R}$ ) is given pointwise + and $\leq ; \mathbb{R}^{S} \in$ Arch.

Theorem 1.3 (4.1 below). If Hypothesis 1.2 holds, then there is an $E C G \leq \mathbb{R}^{S}$ (constructed from $\mathcal{W})$ with $G \notin F(\mathbf{f r} \mathbf{A})$.

Conjecture 1.4. If there is an $E C G \notin F(\boldsymbol{f r} \boldsymbol{A})$, then Hypothesis 1.2 holds.

The rest of this section explains where Question 1.1 comes from, and is technically not required for the proof of Theorem [1.3. Question 1.1] is closed related to issues of representation of Archimedean $l$-structures in lattices

$$
D(Y)=\left\{f \in C(Y,[-\infty,+\infty]) \mid f^{-1} \mathbb{R} \text { dense in } Y\right\} .
$$

Here $Y$ is a Tychonoff space, $[-\infty,+\infty]=\mathbb{R} \cup\{ \pm \infty\}$ with the obvious order and topology, and $D(Y)$ is given the pointwise order. Addition is partially defined by $f_{1}+f_{2}=f_{3}$ means $f_{1}(x)+f_{2}(x)=f_{3}(x)$ when all three are real, and likewise multiplication. This + (respectively, $\cdot)$ is fully defined if and only if each dense cozero set is $C^{*}$-embedded in $Y$ HJ61. This condition defines quasi-F (QF) spaces; Then $D(Y) \in \mathbf{f r} \mathbf{A}$ with the constant function $1_{Y}$, the ring identity.

$Y$ is called extremally (resp. basically) disconnected (ED, resp. BD) if each open (resp. cozero) set has open closure. (See GJ76]). We have $\mathrm{ED} \Rightarrow \mathrm{BD} \Rightarrow \mathrm{QF}$, and $D(Y)$ is an $l$-group which is laterally and conditionally complete ( $\sigma$-complete) if and only if $Y$ is ED (resp., BD). These extend the Nakano-Stone Theorems for $C(X)$. (See [BHJK09]).

In particular, $D(Y)$ is an Arch-object which is EC if and only if $Y$ is BD. (See BH90a]).

By a representation of $G \in \mathbf{A r c h}$ (resp. frA), we mean a one-to-one map $\epsilon: G \rightarrow D(Y)$ for which $\epsilon(G)$ is closed under the partial operations in $D(Y)$ requisite that $\epsilon(G) \in \mathbf{A r c h}$ (resp. frA $\mathbf{A}$ ), and $G \stackrel{\epsilon}{\rightarrow} \epsilon(G)$ is an l-group (resp. l-ring) isomorphism.

A Johnson representation of $G \in$ Arch is a representation $G \leq D(Y)$ (suppressing the $\epsilon$ ) satisfying

$\left(J_{Y}\right) y \notin F$ closed in $Y \Rightarrow \exists g \in G$ with $0<g(y)<+\infty$, and

$\left(J_{\infty}\right) \forall g \in G$, the Čech-Stone extension has $\beta g(\beta Y-Y) \subseteq\{0, \pm \infty\}$.

(It follows that $Y$ is locally compact). We may say " $J$-representation", and indicate such a situation as $G \stackrel{J}{\leq} D(Y)$. It is so-called because:

Theorem 1.5 (Joh62, Joh07]). For each $(G, *) \in$ frA $\mathbf{A}$, there is $G \stackrel{J}{\leq} D(Y)$ with $G$ closed in $D(Y)$ under the partial .

Consider Y locally compact. Let $D_{0}(Y) \equiv\{f \in D(Y) \mid \beta f(\beta Y-Y)=\{0\}\}$ (the functions that vanish at $\infty$ ). If $Y$ is $\mathrm{BD}$, then $D_{0}(Y) \in \mathbf{A r c h}$, is conditionally $\sigma$-complete $($ BH90a, 3.1$)$, and is laterally $\sigma$-complete if and only if either $Y$ is compact (whence $D_{0}(Y)=D(Y)$ ) or $Y$ is not compact and in the one-point compactification $Y \cup\{\alpha\}, \alpha$ is a $P$-point ([BH90a $, \S 4,5)$.

For the nonce, a "good" representation of EC $G$ is an isomorphism $G \approx D_{0}(Y)$, for $Y$ locally compact and $\mathrm{BD}$ (thus, necessarily, $\alpha$ is a $P$-point). 
The following sums up partial results inspiring, and giving four versions of, Question 1.1. Ball's truncations Bal13 will be discussed in $\S 5$.

Theorem 1.6. Suppose $G$ is $E C$.

(1) These are equivalent.

(a) $G \in F(\mathbf{f r} \mathbf{A})$.

(b) $G$ has a Johnson representation.

(c) $G$ has a good representation.

(d) $G$ has a compatible truncation.

(2) If $G$ has any weak unit, these four conditions obtain.

BH90b shows (2): For $G$ with weak unit $e$, there is $X$ compact BD, with $G \approx D(X)$ and $e \mapsto 1_{X}$.

Regarding (1): Johnson's 1.4 shows (a) $\Rightarrow(\mathrm{b})$, and Ball's 5.1 (below) shows (d) $\Rightarrow(\mathrm{b})$ (neither needing " $G$ EC"). From $\S 5,(\mathrm{c}) \Rightarrow(\mathrm{d})$ is clear. BH90a shows (b) $\Rightarrow$ (c), at considerable labor. (c) $\Rightarrow$ (a) is evident.

We mentioned that EC is monoreflective in Arch. In that regard, there is more, as follows.

Let $\mathbf{W}$ be the (non-full) subcategory of Arch with objects $\left(G, e_{G}\right), e_{G}$ is a positive weak unit of $G$, and morphisms $\left(G, e_{G}\right) \stackrel{\varphi}{\rightarrow}\left(H, e_{H}\right)$ the Arch-morphisms with $\varphi\left(e_{G}\right)=e_{H}$. In $\mathbf{W}$, the remarkably canonical Yosida representation has many consequences, which are striking, and food for thought, in comparison with Arch. This representation of a $\left(G, e_{G}\right)$ can be described as the essentially unique $G \leq D(Y G)$ which is a $J$-representation, $Y G$ is compact, and $e_{G} \mapsto 1$. See [HR77].

EC in W has "the same" characterization as in Arch, divisible, laterally and conditionally $\sigma$-complete. It results that $\left(G, e_{G}\right)$ is $\mathbf{W}$-EC if and only if $\left(G, e_{G}\right) \approx$ $\left(D(X), 1_{X}\right)$, for $X$ compact BD. (The $X$ is the $Y G$ above. We alluded to this in Theorem 1.6 (2), above.) Similar to Arch, W-EC is the smallest monoreflective subcategory of $\mathbf{W}$. (This information is in BH90b, BH90a.)

Let $\beta^{\mathbf{A r c h}}$ and $\beta^{\mathbf{W}}$ denote their "EC-monoreflection" functors. In BH90a, we find a concrete calculation/representation/description of $G \leq \beta^{\mathbf{W}} G$ (suppressing units), in terms of $G \leq D(Y G)$. In [BHJK05], we found a quite similar description of $G \leq \beta^{\operatorname{Arch}} G$, for $G=C_{K}(Y)$, or $C_{0}(Y)$ the functions of compact support, or vanishing at $\infty$, on locally compact $Y$, and these $\beta^{\text {Arch }} G$ have the features in Theorem [1.6 (1). Note that $C_{K}(Y)$ and $C_{0}(Y)$ are, as presented, in $J$-representation. One imagines that the descriptions of these $\beta^{\operatorname{Arch}} G$ alluded to above can be carried through for any $G$ in a $J$-representation, and that if $(G, *) \in \mathbf{f r} \mathbf{A}$, then there is $*^{\prime}$ on $\beta^{\operatorname{Arch}} G$ with $(G, *) \leq\left(\beta^{\operatorname{Arch}} G, *^{\prime}\right)$ in frA.

\section{Some EPICOMPLETE $l$-GROUPS}

$S$ is a set of cardinal $|S|=\aleph_{1}$.

$\mathcal{W}$ is an uncountable family of uncountable subsets of $S$ for which, if $A, B \in \mathcal{W}$ and $A \neq B$, then $|A \cap B| \leq \aleph_{0}$. (There are no further assumptions on $\mathcal{W}$ in this section.) $\mathbb{R}^{S}$ carries pointwise + and $\leq ; \mathbb{R}^{S} \in$ Arch. $P=\left\{f \in \mathbb{R}^{S} \mid f(x)>0 \forall x \in\right.$ $S\}$. This is the set of positive weak units in $\mathbb{R}^{S}$.

$\mathcal{W} \stackrel{\gamma}{\rightarrow} P$ is any function.

In this section, we construct epicomplete $G(\mathcal{W}, \gamma) \leq \mathbb{R}^{S}$. The construction is modeled on the elaboration in HJ10 of the example in CM90. In $\S 4$, we will specify the $(\mathcal{W}, \gamma)$ to make $G(\mathcal{W}, \gamma) \notin F(\mathbf{f r} \mathbf{A})$. 
We adopt the following notations.

For $U, V \subseteq S, U \stackrel{*}{\subseteq} V$ means there is countable $T$ with $U \subseteq V \cup T$, and $U \stackrel{*}{=} V$ means $U \stackrel{*}{\subseteq} V$ and $V \stackrel{*}{\subseteq} U$. (For $A \neq B$ in $\mathcal{W}, A \cap B \stackrel{*}{=} \emptyset$.)

For $f, g \in \mathbb{R}^{S}, f \stackrel{*}{\leq} g$ means there is countable $T$ with $f(x) \leq g(x)$, for $x \in S-T$, and $f \stackrel{*}{=} g$ means $f \stackrel{*}{\leq} g$ and $g \stackrel{*}{\leq} f$.

For $\mathcal{A} \subseteq \mathcal{W}$ with $|\mathcal{A}| \leq \aleph_{0}$, let $c(\mathcal{A})=\cup\{A \cap B \mid A \neq B$ in $\mathcal{A}\}$. Observe $|c(\mathcal{A})| \leq \aleph_{0}$.

For $A \in \mathcal{A}$, let $\dot{A}=A-c(\mathcal{A})$. Let $\dot{\mathcal{A}}=\{\dot{A} \mid A \in \mathcal{A}\}$. Observe $\dot{A} \cap \dot{B}=\emptyset$ for $A \neq B$ in $\mathcal{A}$.

Again with $\mathcal{A} \subseteq \mathcal{W}$ and $|\mathcal{A}| \leq \aleph_{0}$, let $r: S \rightarrow \mathbb{R}$ have the properties: for $x \notin \bigcup_{\mathcal{A}} \dot{A}, r(x)=0$; for $A \in \mathcal{A}, r \mid \dot{A}$ is constant, denoted $r_{A}$.

For $U \subseteq S$, let $\chi_{U}$ be the characteristic function of $U$.

Definition 2.1. For $(\mathcal{A}, r)$ as above, let

$$
u(\mathcal{A}, r) \equiv \sum_{\mathcal{A}} r_{A} \chi_{\dot{A}} \gamma(A) .
$$

(Here, the $\sum$ is pointwise in $\mathbb{R}^{S}$. The supports of the summands are disjoint, so the $\sum_{\mathcal{A}}$ makes sense.)

$$
G(\mathcal{W}, \gamma) \equiv\left\{g \in \mathbb{R}^{S} \mid \text { there is }(\mathcal{A}, r) \text { with } g \stackrel{*}{=} u(\mathcal{A}, r)\right\} .
$$

Theorem 2.2. $G(\mathcal{W}, \gamma)$ is a sub-l-group of $\mathbb{R}^{S}$, without weak units, and is epicomplete.

Proof of 2.2. In the following, $\mathcal{A}, \mathcal{B}, \ldots$ always denote countable subsets of $\mathcal{W}$.

The proof has a number of steps. The first may not be completely necessary, but is articulated to avoid confusion.

Lemma 2.3. The following are equivalent.

(1) $\mathcal{A} \subseteq \mathcal{B}$.

(2) $\bigcup_{\mathcal{A}} A \subseteq \bigcup_{\mathcal{B}} B$.

(3) $\bigcup_{\mathcal{A}} \dot{A} \subseteq \bigcup_{\mathcal{B}} \dot{B}$.
(2') $\bigcup_{\mathcal{A}} A \stackrel{*}{\subseteq} \bigcup_{\mathcal{B}} B$.

(3') $\cup_{\mathcal{A}} \dot{A} \stackrel{*}{\subseteq} \bigcup_{\mathcal{B}} \dot{B}$.

Proof. (Note $(3)$ says $\bigcup_{\mathcal{A}}(A-c(\mathcal{A})) \subseteq \bigcup_{\mathcal{B}}(B-c(\mathcal{B}))$, and $\mathcal{A} \subseteq \mathcal{B} \Rightarrow c(\mathcal{A}) \supseteq c(\mathcal{B})$.)

These implications are obvious: $(1) \Rightarrow(2) \Rightarrow\left(2^{\prime}\right) ;(3) \Rightarrow\left(3^{\prime}\right)$. Also, for any $\mathcal{A}$, $\bigcup_{\mathcal{A}} A=\bigcup_{\mathcal{A}} \dot{A} \cup c(A)$; so $\left(3^{\prime}\right) \Rightarrow\left(2^{\prime}\right)$.

$\left(2^{\prime}\right) \Rightarrow(1)$. Suppose (1) fails, with $A \in \mathcal{A}-\mathcal{B}$. Thus, $\forall B \in \mathcal{B}, A \cap B$ is countable, and so is $A \cap \bigcup_{\mathcal{B}} B=\bigcup_{\mathcal{B}}(A \cap B)$ (since $\mathcal{B}$ is countable). Then, for any countable $T, A \cap\left(\bigcup_{\mathcal{B}} B \cup T\right)$ is countable, so $A \nsubseteq \bigcup_{\mathcal{B}} B \cup T$ (since $\left.|A| \geq \aleph_{1}\right)$. So (2') fails.

$(1) \Rightarrow(3)$. Suppose $A \in \mathcal{A}$. Then, $A-c(\mathcal{A}) \stackrel{*}{=} A-c(\mathcal{B})$, so $\bigcup_{\mathcal{A}}(A-c(\mathcal{A})) \stackrel{*}{=}$ $\bigcup_{\mathcal{A}}(A-c(\mathcal{B})) \subseteq \bigcup_{\mathcal{B}}(B-c(\mathcal{B}))$ (the last $\subseteq$ because $\left.\mathcal{A} \subseteq \mathcal{B}\right)$.

In the following, $G(\mathcal{W}, \gamma)$ is denoted $G$. We shall prove below that $G \leq \mathbb{R}^{S}$ (i.e., is a sub-l-group,) and $G$ is EC.

Granted $G \leq \mathbb{R}^{S}, G$ has no weak units:

If $g \in G^{+}$has $g^{\perp}=\{0\}$, then $\forall x \in S, g \wedge \chi_{x}>0$, which means $g(x)>0$, so $\operatorname{coz} g=S$. But $g \stackrel{*}{=} u(\mathcal{A}, r)$, so $S=\operatorname{coz} g \stackrel{*}{=} \operatorname{coz} u(\mathcal{A}, r) \subseteq \bigcup_{\mathcal{A}} \dot{A}$. But $|\mathcal{A}| \leq \aleph_{0}$ 
and $\mathcal{W}$ is uncountable, so there is $B \in \mathcal{W}$ with $B \notin \mathcal{A}$, thus $\left|B-\bigcup_{\mathcal{A}} \dot{A}\right| \geq \aleph_{1}$. Contradiction.

Lemma 2.4. Suppose $\mathcal{A} \subseteq \mathcal{B}$. Given $u(\mathcal{A}, r)$, there is $\bar{r}$ with $u(\mathcal{B}, \bar{r}) \stackrel{*}{=} u(\mathcal{A}, r)$ as follows.

$\bar{r}(x)=0$ if $x \notin \bigcup_{\mathcal{B}} \dot{B}$ or if $x \in \dot{B}$ for $B \in \mathcal{B}-\mathcal{A}$.

If $A \in \mathcal{A}, \bar{r}|(A-c(\mathcal{B}))|=r_{A}$ (the constant value of $r$ on $A-c(\mathcal{A})$; i.e. $\bar{r}_{A}=r_{A}$ ).

Proof. $\bigcup_{\mathcal{A}}(A-c(\mathcal{B})) \stackrel{*}{=} \bigcup_{\mathcal{A}}(A-c(\mathcal{A}))$, and $\bar{r}=r$ on the left set.

Corollary 2.5. Suppose given $u\left(\mathcal{A}_{n}, r_{n}\right)$ for $n=1,2$ or $n=1,2, \ldots$ Let $\mathcal{B}=$ $\bigcup_{n} \mathcal{A}_{n}$. Apply Lemma 2.4 to obtain $u\left(\mathcal{B}, \bar{r}_{n}\right) \stackrel{*}{=} u\left(\mathcal{A}_{n}, r_{n}\right)$ for each $n$.

Corollary 2.6. $G \subseteq \mathbb{R}^{S}$ is closed under the inherited $+,-, \vee, \wedge$. I.e., $G \leq \mathbb{R}^{S}$.

Proof. Let $\otimes \in\{+,-, \vee, \wedge\}$. Let $g_{1}, g_{2} \in G$, as $g_{\dot{n}} \stackrel{*}{=} u\left(\mathcal{A}_{\dot{n}}, r_{\dot{n}}\right)$. Take $\mathcal{B}=\mathcal{A}_{1} \cup \mathcal{A}_{2}$ and apply Corollary 2.5 to get $u\left(\mathcal{B}, \bar{r}_{\dot{n}}\right) \stackrel{*}{=} u\left(\mathcal{A}_{n}, r_{n}\right)$. Let $r=\bar{r}_{1} \otimes \bar{r}_{2}$. Observe that $\mathcal{B}=\left(\mathcal{A}_{1} \cap \mathcal{A}_{2}\right) \cup\left(\mathcal{A}_{1}-\mathcal{A}_{2}\right) \cup\left(\mathcal{A}_{2}-\mathcal{A}_{1}\right)$; it follows that $\bar{r} \mid \dot{B}$ is constant $\forall B \in \mathcal{B}$.

For countable suprema, we proceed as follows.

Lemma 2.7. Suppose $\left\{g_{n}\right\}_{\mathbb{N}} \subseteq G^{+}$and in $\mathbb{R}^{S}, \vee g_{n}$ exists. (This $\vee$ is pointwise, and saying it exists just means $\left\{g_{n}\right\}$ is bounded above in $\mathbb{R}^{S}$, i.e., $\forall x,\left\{g_{n}(x)\right\}_{\mathbb{N}}$ is bounded above in $\mathbb{R}$.) Call this $\vee g_{n}$ as $g$.

Then, $g \in G$ and therefore $g=\vee^{G} g_{n}$.

Proof. Express each $g_{n}$ as $g_{n} \stackrel{*}{=} u\left(\mathcal{A}_{n}, r_{n}\right)$; note $r_{n} \geq 0$. Let $\mathcal{B}=\bigcup_{n} \mathcal{A}_{n}$, and apply 2.5 $\forall n$, getting $u\left(\mathcal{B}, \bar{r}_{n}\right) \stackrel{*}{=} u\left(\mathcal{A}_{n}, r_{n}\right)$ (with $\bar{r}_{n}=0$ on $D-\bigcup_{\mathcal{B}} \dot{B}$, and $\bar{r}_{n} \geq 0$ ).

Since $g_{n} \leq g$ and $g_{n}=u\left(\mathcal{B}, \bar{r}_{n}\right)$ except on a countable $T_{n}, u\left(\mathcal{B}, \bar{r}_{n}\right) \leq g$ except on $T_{n}$. We have successively: $\forall n u\left(\mathcal{B}, \bar{r}_{n}\right) \leq g$ except on the countable $T=\bigcup_{n} T_{n}$; $\forall n \bar{r}_{n} \chi_{\dot{B}} \gamma(B) \leq u\left(\mathcal{B}, \bar{r}_{n}\right) \leq g$ except on $T \forall B \in \mathcal{B} ; \forall n \forall B$, on $\dot{B}$ except on $T$, $\bar{r}_{n} \gamma(B) \leq g$, or $\bar{r}_{n} \leq \frac{g}{\gamma(B)}$.

Now define $r: S \rightarrow \mathbb{R} \equiv 0$ on $S-\bigcup_{\mathcal{B}} \dot{B}$, and for $B \in \mathcal{B}$, on $\dot{B} r \equiv \vee_{n} \bar{r}_{n}$. (This is pointwise $\vee$ of the $\bar{r}_{n}$ which are constant on $\dot{B}$, so it is the $\vee$ in $\mathbb{R}$ of those constant values " $\left(\bar{r}_{n}\right)_{B}$ ".)

Then, $g \stackrel{*}{=} u(\mathcal{B}, r)$ :

Clearly, each is 0 on $S-\bigcup_{\mathcal{B}} \dot{B}$ except for $T$.

For $B \in \mathcal{B}$ and $x \in \dot{B}-T$, we have $u\left(\mathcal{B}, \bar{r}_{n}(x)\right)=\bar{r}_{n} \gamma(B)(x)=g_{n}(x)$, so $r(x) \gamma(\mathcal{B})(x)=\left(\vee \bar{r}_{n}(x)\right) \gamma(B)(x)=\vee\left(\bar{r}_{n} \gamma(B)\right)(x)=\vee g_{n}(x)=g(x)$.

Note, Corollary 2.6] says $\left\{g_{n}\right\} \subseteq G^{+}$is bounded above in $G$ if and only if in $\mathbb{R}^{S}$.

Corollary 2.8. $G$ is EC, i.e., divisible, laterally $\sigma$-complete, and conditionally $\sigma$-complete.

Proof. Divisibility is obvious. If $\left\{g_{n}\right\}$ is disjoint (resp., bounded above in $G$ ), then in $\mathbb{R}^{S}, \vee g$ exists because $\mathbb{R}^{S}$ is laterally $\sigma$-complete (resp., conditionally $\sigma$-complete). Apply Corollary 2.6.

Theorem 2.2 is proved. 


\section{Essential completions and compatible multiplications}

We explain essential completions, and note that the $G(\mathcal{W}, \gamma) \leq \mathbb{R}^{S}$ (in Theorem 2.2) is one (Corollary 3.3). The point is that, in general, " $G \in F(\mathbf{f r} \mathbf{A})$ " can be recognized in terms of any particular essential completion of $G$ (Corollaries 3.5 and 3.6).

The basic framework is due to Bernau Ber65] and Conrad [Con71,Con74]. Embellishments are added for clarity, and to the present purpose.

Let $X$ be a compact extremally disconnected (ED) space; so that $D(X) \in$ Arch and $(D(X), \cdot) \in \mathbf{f r} \mathbf{A}($ see $\S 1)$.

Suppose $X \stackrel{\tau}{\leftarrow} X$ is a homeomorphism. Define $D(X) \stackrel{\bar{\tau}}{\rightarrow} D(X)$ as $\bar{\tau}(f)=f \circ \tau$. Suppose $d \in P \equiv$ the set of positive weak units of $D(X)\left(d \in P \equiv d^{\perp}=\{0\}\right.$ if and only if $\operatorname{coz} d \equiv\{x \mid d(x) \neq 0\}$ is dense in $X)$. Define $D(X) \stackrel{\bar{d}}{\rightarrow} D(X)$ as $d(f)=d \cdot f$ (pointwise multiplication in $D(X))$. Note that $d^{-1} \in P .\left(d^{-1}\right.$ is the Cech-Stone extension of $\frac{1}{d} \mid \operatorname{coz} d$.) Define $\otimes_{d}$ on $D(X)$ as $f_{1} \otimes_{d} f_{2} \equiv d^{-1} f_{1} f_{2}$.

In the rest of this section, " $d$ " denotes a $d \in P$.

\section{Theorem 3.1.}

(1) The frA-isomorphisms of $(D(X), \cdot)$ are exactly the $\bar{\tau}$.

(2) Each $\bar{d}$ is an l-group isomorphism of $D(X)$.

(3) The l-group isomorphisms of $D(X)$ are exactly the $\bar{d} \bar{\tau}$.

(4) $\left(D(X), \otimes_{d}\right) \in \mathbf{f r A}$ with identity $d$ and $(D(X), \cdot) \stackrel{\bar{d}}{\rightarrow}\left(D(X), \otimes_{d}\right)$ is a frAisomorphism.

All of Theorem 3.1 was certainly known to Bernau and Conrad, but we note: In (1), that $\bar{\tau}$ is a frA-isomorphism is easy, and the converse is a special case of HR77. (2) is easy. In (3), that $\bar{d} \bar{\tau}$ is an l-group isomorphism follows from (1) and (2), and the converse is a special case of [BH09. (4) is (now easy and) completely recognized in Con74, $\S 2$.

In a general category: An essential monic is a monic $\varphi$ for which $\psi \varphi$ monic implies $\psi$ monic. $E$ is essentially complete if $E \stackrel{\varphi}{\rightarrow}$ • essential monic implies $\varphi$ an isomorphism. An essential completion (of $G$ ) is a $G \stackrel{\varphi}{\rightarrow} E$ with $\varphi$ essential monic and $E$ essentially complete. (If the category lacks injective hulls, this notion substitutes.)

In Arch, monic means one-to-one, and (suppressing label) $G \leq H$ is essential if and only if $G$ is large in $H$, meaning: if $0<h \in H$, there are $0<g \in G$ and $n \in \mathbb{N}$ with $g \leq n h$.

The following is all in Arch.

\section{Theorem 3.2.}

(1) $E$ is essentially complete if and only if $E \approx$ some $D(X)$ for $X$ compact $E D$ if and only if $E$ is divisible, laterally complete, and conditionally complete.

(2) For any $G$, there is an essential completion $G \stackrel{\varphi}{\leq} D(X)$.

(3) For every two essential completions, $G \stackrel{\varphi_{i}}{\leq} D(X)$, there is a unique l-group isomorphism $\mu$ with $\mu \varphi_{1}=\varphi_{2}$. (Note $\mu=\bar{d} \bar{\tau}$ as in Theorem 3.1.)

In Theorem 3.2 (1) is due to Conrad Con71. (2) and (3) combine (1) with facts about Bernau's representation Ber65. 
Corollary 3.3. In $2.2, G(\mathcal{W}, \gamma) \leq \mathbb{R}^{S}$ is an essential completion. (The $X$ in Theorem 3.2 is $\beta S ; \mathbb{R}^{S} \approx D(\beta S)$.)

Proof. $\mathbb{R}^{S}$ is visibly divisible, laterally and conditionally complete (or, referring to Theorem 3.2, $\beta S$ is ED). $G(\mathcal{W}, \gamma)$ is large in $\mathbb{R}^{S}$ : If $f>0$, there is $x$ with $f(x)>0$, so there is $n$ with $n f(x)>1$, so $0<\chi_{x} \leq n f$, and $\chi_{x} \in G(\mathcal{W}, \gamma)$.

Theorem 3.4. If $(G, *) \in$ frA $\mathbf{A}$, then there is $(G, *) \stackrel{\psi}{\leq}(D(X), \cdot)$ in $\mathbf{f r} \mathbf{A}$ with $G \stackrel{F \psi}{\leq} D(X)$ an essential completion.

Bernau shows Theorem 3.4 for his representation Ber65, which Conrad identified as an essential completion [Con71]. (Another proof of Theorem 3.4 is indicated after Remark [5.3])

Corollary 3.5. Suppose $G \stackrel{\eta}{\leq} D(X)$ is any essential completion.

(1) If there is $d$ such that $\eta(G)$ is closed under $\otimes_{d}$ in $D(X)$, then $\left(\eta(G), \otimes_{d}\right) \in$ frA and $G \in F($ fr $\mathbf{A})$.

(2) If there is $*$ with $(G, *) \in \mathbf{f r} \mathbf{A}$ (i.e., $G \in F(\mathbf{f r} \mathbf{A})$ ), then there is $d$ with $(G, *) \approx\left(\eta(G), \otimes_{d}\right)$.

Proof. (1) is obvious.

(2). We use Theorems 3.1, 3.2, and 3.4, Consider

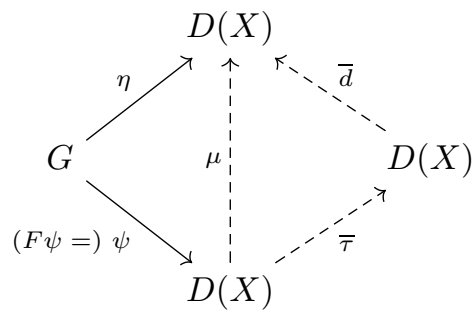

where $\eta$ is the given, $(G, *) \stackrel{\psi}{\leq}(D(X), \cdot)$ is from Theorem 3.4, and $\mu$ with $\mu \psi=\eta$ comes from Theorem $3.2(3)$, and $\mu=\bar{d} \bar{\tau}$ by Theorem 3.1 .

The claim is that $\eta(G)$ is closed under $\otimes_{d}$, as $d^{-1} \eta\left(g_{1}\right) \eta\left(g_{2}\right)=\eta\left(g_{1} * g_{2}\right)$ :

$$
\begin{aligned}
\eta\left(g_{1} * g_{2}\right) & =\mu \psi\left(g_{1} * g_{2}\right)=\mu\left(\psi\left(g_{1}\right) \cdot \psi\left(g_{2}\right)\right) \\
& =\bar{d} \bar{\tau}\left(\psi\left(g_{1}\right) \cdot \psi\left(g_{2}\right)\right)=\bar{d}\left(\bar{\tau} \psi\left(g_{1}\right) \cdot \bar{\tau} \psi\left(g_{2}\right)\right) \\
& =d \cdot \bar{\tau} \psi\left(g_{1}\right) \cdot \bar{\tau} \psi\left(g_{2}\right)=d \cdot \bar{\tau} \psi\left(g_{1}\right) \cdot\left(d^{-1} d\right) \cdot \bar{\tau} \psi\left(g_{2}\right) \\
& =d^{-1} \cdot d \bar{\tau} \psi\left(g_{1}\right) \cdot d \bar{\tau} \psi\left(g_{2}\right) \\
& =d^{-1} \cdot \bar{d} \bar{\tau} \psi\left(g_{1}\right) \cdot \bar{d} \bar{\tau} \psi\left(g_{2}\right)=d^{-1} \cdot \mu \psi\left(g_{1}\right) \cdot \mu \psi\left(g_{2}\right) \\
& =d^{-1} \cdot \eta\left(g_{1}\right) \cdot \eta\left(g_{2}\right) .
\end{aligned}
$$

Corollary 3.5 is, more-or-less, in Con74, $§ 2$.

Corollary 3.6. Suppose $G \stackrel{\eta}{\leq} D(X)$ is any essential completion.

(1) $G \in F(\mathbf{f r} \mathbf{A})$ if and only if there is $d$ such that $\eta(G)$ is closed under $\otimes_{d}$ in $D(X)$. 
(2) $G \notin F(\mathbf{f r} \mathbf{A})$ if and only if $\forall p \in P, \exists g_{1}, g_{2} \in G$ with $p \cdot \eta\left(g_{1}\right) \cdot \eta\left(g_{2}\right) \notin \eta(G)$. Proof. (1) restates Corollary 3.5. (2) is the same as (1) using $p=d^{\dashv}$.

Note. If $G$ is divisible, the condition in Corollary 3.6 (2) is equivalent to: $\forall p, \exists g$ with $p \cdot \eta(g)^{2} \notin \eta(G)$ (because $\left.(x+y)^{2}=x^{2}+2 x y+y^{2}\right)$.

\section{Epicomplete $G \notin F(\mathbf{f r} \mathbf{A})$.}

Such $G$ will be of the form $G(\mathcal{W}, \gamma) \leq \mathbb{R}^{S}$ as in $\S 2$. The items $S, \mathcal{W}, P, \ldots$ are as in $\S 2$.

Theorem 4.1. If $|\mathcal{W}|=2^{\aleph_{1}}$, then there is $\mathcal{W} \stackrel{\delta}{\rightarrow} P$ for which $G(\mathcal{W}, \delta) \notin F(\mathbf{f r} \mathbf{A})$.

Combining Theorem 4.1 with Theorem 2.2. and with other information in $\S 1$, gives the full statement of Theorem 1.3

The definition of the $\delta$ requires a lemma.

Lemma 4.2. Suppose $A \subseteq S$ with $|A|=\aleph_{1}$, and suppose $f \in P$. Then there is $h \in P$ for which

(i) $h(x) \geq f(x)$ for each $x \in S$, and

(ii) for any countable $T \subseteq S$ and any $r \in \mathbb{R}, h|(A-T) \neq r f|(A-T)$.

Proof. Given the $A$ and $f$, we define separately $h \mid(S-A)$ and $h \mid A$ :

$h|(S-A) \equiv(f+1)|(S-A)$.

If there is no countable $T$ with $f \mid(A-T)$ constant, then $\forall$ such $T,|f(A-T)| \geq 2$, and again define $h|A=(f+1)| A$. (If $(f+1)|(A-T)=r f|(A-T)$, then $\forall x \in A-T$, $f(x)=\frac{1}{r-1}$, contradicting $|f(A-T)| \geq 2$.)

Suppose there is countable $T$ with $f \mid(A-T)$ constant, take one-to-one $A \stackrel{\beta}{\rightarrow} \mathbb{R}$, and define $h|A \equiv(f+\beta)| A$. (If there were countable $T^{\prime}$ and $r$ with $h \mid\left(A-T^{\prime}\right)=$ $r f \mid\left(A-T^{\prime}\right)$, then also $h=r f$ on $A-\left(T^{\prime} \cup T\right)$ where $f$ is constant, say $r_{0}$. Then, on $A-\left(T^{\prime} \cup T\right), \beta=(r-1) r_{0}$ constant, contradicting $\beta$ one-to-one.)

Proof of Theorem 4.1. We shall define $\delta$ using Lemma 4.2, which gives $G(\mathcal{W}, \delta) \leq$ $\mathbb{R}^{S}$ as in Theorem [2.2, and then verify 3.6 to get $G(\mathcal{W}, \delta) \notin F(\operatorname{fr} \mathbf{A})$. Since $|\mathcal{W}|=$ $2^{\aleph_{1}}$, there is a bijection $\mathcal{W} \stackrel{\alpha}{\leftarrow} P$; for each $A \in \mathcal{W}$, there is $v \in P$ with $A=\alpha(v)$. Apply Lemma 4.2 to $f=1 / v$, producing $h \equiv \delta(A)$ for which

(ii) $\forall T, r, h\left|(A-T) \neq \frac{r}{v}\right|(A-T)$.

Theorem 2.2 applies to $G \equiv G(\mathcal{W}, \delta) \leq \mathbb{R}^{S}$, which is an essential completion (Corollary 3.3). We use Corollary 3.6 (suppressing the $\eta$ there). We claim: $\forall v \in P$, $\exists g \in G$ with $v g^{2} \notin G$. Take $v \in P$, let $A=\alpha(v)$, and let $g=\chi_{A} \gamma(A)=\chi_{A} h, h$ satisfying (ii) above. (This $g$ is $u\left(\{A\}, \chi_{A}\right)$.) Then, $g^{2}=\chi_{A} h^{2}$ and $v g^{2}=v \chi_{A} h^{2}$.

Suppose (towards contradiction) that $v \chi_{A} h^{2} \in G$. Then, $v \chi_{A} h^{2} \stackrel{*}{=} u(\mathcal{B}, r)$ for some $(\mathcal{B}, r)$, and using Lemma 2.4 we can suppose $A \in \mathcal{B}$ (by replacing $\mathcal{B}$ by $\mathcal{B} \cup\{A\}$ ). For $\dot{A}=A-c(\mathcal{B})$, we then have $v \chi_{\dot{A}} h^{2}=r \chi_{\dot{A}} \gamma(A)=r \chi_{\dot{A}} h$ (from the form of $u(\mathcal{B}, r)$ ). Cancelling an $h$ gives $v \chi_{\dot{A}} h=r \chi_{\dot{A}}$, whence $h=\frac{r}{v}$ on $A-c(\mathcal{B})$, contradicting (ii) above.

\section{Compatible truncation}

Suppose $G \in \mathbf{A r c h}$, and $e$ is a positive weak unit of $G$. Then $G^{+}$is closed under the "truncation" $g \mapsto e \wedge g \equiv t_{e}(g)$. Ball [Bal13] has viewed this as a unary 
operation, abstracted (axiomatized) its features, and defined the category AT of Archimedean "truncs": The $(G, t), t: G^{+} \rightarrow G^{+}$satisfying his axioms (which we need not list), with morphisms $(G, t) \stackrel{\varphi}{\rightarrow}\left(G^{\prime}, t^{\prime}\right)$ the l-group morphisms with $\varphi(t(g))=t^{\prime}(\varphi(g)) \forall g \in G^{+}$.

Let Arch $\stackrel{F}{\leftarrow}$ AT be the forgetful functor.

Note that any $D(Y)$ (not necessarily an l-group) has $f \wedge 1_{Y} \in D(Y) \forall f \geq 0$, and $f \in D_{0}(Y)$ implies $f \wedge 1_{Y} \in D_{0}(Y)$.

Here is Ball's companion to Johnson's Theorem 1.4.

Theorem 5.1 ([Bal13]). For each $(G, t) \in \mathbf{A T}$, there is a J-representation $G \stackrel{\alpha}{\leq}$ $D_{0}(Y)$ with $\alpha(t(g))=1_{Y} \wedge \alpha(g)$ for each $g \in G^{+}$.

At this point, we see that for $\mathrm{EC} G, G \in F(\mathbf{A T})$ if and only if $G \in F(\mathbf{f r} \mathbf{A})$, in consequence of Johnson's 1.4, Ball's 5.1, and the long argument in BHJK09. that if EC $G$ has any $J$-representation, then $G \approx D(K, p)$, the latter evidently in $F(\mathbf{f r} \mathbf{A}) \cap F(\mathbf{A T})$. We described this in $\S 1$.

Thus, the $G(\mathcal{W}, \delta)$ in Theorem 4.1 has $G(\mathcal{W}, \delta) \notin F(\mathbf{A T})$.

We shall directly produce other $G(\mathcal{W}, \tau) \leq \mathbb{R}^{S}$ with $G(\mathcal{W}, \tau) \notin F(\mathbf{A T})$, by arguing in parallel to $\S 3$ and Theorem 4.1. We do this in the hope of ultimately understanding why, on a $G \in \mathbf{A r c h}$, truncations and frA-multiplications behave so similarly: we do not yet.

Corollary 5.2. Suppose $(G, t) \in \mathbf{A T}$.

(1) There is $H \in \mathbf{A r c h}$ with a weak unit e, and an embedding $(G, t) \stackrel{\beta}{\leq}\left(H, t_{\beta}\right)$ (i.e., qua $\mathbf{A T}$ ) with $G \stackrel{F \beta}{\leq} H$ essential.

(2) There is $X$ compact $E D$ and $(G, t) \stackrel{\psi}{\leq}\left(D(X), t_{1}\right)\left(1=1_{X}\right)$ with $G \stackrel{F \psi}{\leq} D(X)$ an essential completion.

Proof.

(1) Take $G \stackrel{\alpha}{\leq} D_{0}(Y)$ per Theorem 5.1, let $H=j m\left(\alpha(G)+\mathbb{Z} \cdot 1_{Y}\right)$. (Here, $m(\bullet)$ is the collection of all finite meets from $(\bullet)$, and likewise $j(\bullet)$ for joins.) Then, $H \in \mathbf{A r c h}$, and $e=1_{Y}$ is a weak unit. (See [HJ10, §2.) Take $e=1_{Y}$, and $\beta$ the codomain restriction of the function $\alpha . F \beta$ is essential because $\alpha$ is a Johnson representation.

(2) With $(G, t) \stackrel{\beta}{\leq}(H, e)$ as in $(1)$, take an essential completion $H \stackrel{\gamma}{\leq} D(X)$, for which $\beta(e)=1_{X}$. Then $\psi=\gamma \beta$.

Remark 5.3. (2) also can be proved from the $G \stackrel{\alpha}{\leq} D_{0}(Y)$ in Theorem 5.1 and some topology, like this. Let $\beta Y \stackrel{a}{\leftarrow} a \beta Y \equiv X$ be the ED cover (absolute, Gleason cover, projective cover) of the Čech-Stone compactification. We have $D(Y) \approx D(\beta Y) \stackrel{\bar{a}}{\rightarrow}$ $D(X)$ as $\bar{a}(f)=f \circ a$, which produces $G \stackrel{\psi}{\leq} D(X)$ as $g \mapsto \alpha(g) \mapsto \beta \alpha(g) \mapsto \bar{a}(\beta \alpha(g))$. The embedding is essential because $a$ is irreducible.

In exactly the same way, Bernau's Theorem 3.4 can be proved from Johnson's Theorem 1.4. 
In the following, $X$ is compact $\mathrm{ED}$, and $P$ is the set of positive weak units of $D(X)$, and " $d$ " always denotes a $d \in P$. Given $d$, let $t_{d}(f) \equiv d \wedge f\left(f \in D(X)^{+}\right)$. Recall $D(X) \stackrel{\bar{d}}{\rightarrow} D(X)$ is $\bar{d}(f)=d f$, and $\bar{d}\left(1_{X}\right)=d 1_{X}=d$.

Theorem 5.4. Suppose $G \stackrel{\eta}{\leq} D(X)$ is any essential completion.

(a) If there is $d$ such that $\eta(G)^{+}$is closed under $t_{d}$, then $\left(\eta(G), t_{d}\right) \in \mathbf{A T}$ and $G \in F(\mathbf{A T})$.

(b) If there is $t$ with $(G, t) \in \mathbf{A T}$ (i.e., $G \in F(\mathbf{A T})$ ), then there is d with $(G, t) \approx\left(\eta(G), t_{d}\right)$.

Proof. (a) is obvious, and (b) follows from (a) as in Theorem 3.4, but more simply: Consider

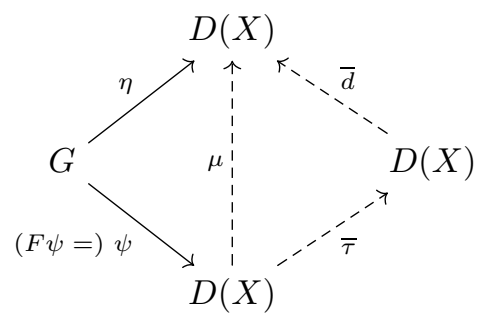

where $\eta$ is the given, $(G, t) \stackrel{\psi}{\leq}\left(D(X), t_{1}\right)$ is from 5.3 (b) $\left(1=1_{X}\right), \mu$ with $\mu \psi=\eta$ comes from Theorem 3.2 (3), and $\mu=\bar{d} \bar{\tau}$ by Theorem 3.1. Note that $\mu(1)=$ $\bar{d} \bar{\tau}(1)=\bar{d}(1)=d$.

The claim is that $\eta(G)^{+}$is closed under $t_{d}$, as $d \wedge \eta(g)=\eta(t(g))$ :

$$
\eta(t(g))=\mu \psi(t(g))=\mu(1 \wedge \psi(g))=\mu(1) \wedge \mu \psi(g)=d \wedge \eta(g) .
$$

Corollary 5.5. Suppose $G \stackrel{\eta}{\leq} D(X)$ is any essential completion. $G \notin F(\mathbf{A T})$ if and only if $\forall d \in P, \exists g \in G^{+}$with $d \wedge \eta(g) \notin \eta(G)$.

Here is the advertised companion to Theorem 4.1. (The $\tau$ appearing has nothing to do with the $\tau$ in $\S 3$ and the proof of Theorem [5.4.)

Theorem 5.6. If $|\mathcal{W}|=2^{\aleph_{1}}$, then there is $\mathcal{W} \stackrel{\tau}{\rightarrow} P$ for which $G(\mathcal{W}, \tau) \notin F(\mathbf{A T})$.

Proof. As in Theorem 4.1, take any bijection $\mathcal{W} \stackrel{\alpha}{\leftarrow} P$; for each $A \in \mathcal{W}$, there is $d \in P$ with $A=\alpha(d)$. Apply Lemma 4.2 exactly as stated to $f=d$, producing $h \equiv \tau(A)$ for which

(i) $h(x) \geq d(x) \forall x \in S$, and

(ii) $\forall$ countable $T \subseteq S, \forall r \in \mathbb{R}, h|(A-T) \neq r d|(A-T)$.

Theorem 2.2 applies to $G \equiv G(\mathcal{W}, \tau) \leq \mathbb{R}^{S}$, which is an essential completion as in Corollary 5.5 (suppressing the $\eta$ there). We claim: $\forall d \in P, \exists g \in G$ with $d \wedge g \notin G$.

Take $d \in P$, let $A=\alpha(d)$, and let $g=\chi_{A} \tau(A)=\chi_{A} h$, with $h$ as above. (This $g$ is $u\left(\{A\}, \chi_{A}\right)$.) Since $h \geq d, d \wedge g=d \wedge \chi_{A} h=d \chi_{A}$.

Suppose (toward contradiction) that $d \chi_{A} \in G$. Then, $d \chi_{A} \stackrel{*}{=} u(\mathcal{B}, r)$ for some $(\mathcal{B}, r)$, and we can suppose $A \in \mathcal{B}$. For $\dot{A}=A-c(\mathcal{B})$, we then have $d \chi_{\dot{A}}=r_{A} \chi_{\dot{A}} h$, which means $h=\frac{1}{r_{A}} d$ on $A-c(\mathcal{B})$, contradicting (ii) above. 


\section{ACKnowledgements}

The author thanks Philip Scowcroft for discussions elucidating the situation of this paper; and also thanks Rick Ball for alerting him to Bal13.

\section{REFERENCES}

[Bal13] Richard N. Ball, Truncated abelian lattice-ordered groups I: The pointed (Yosida) representation, Topology Appl. 162 (2014), 43-65, DOI 10.1016/j.topol.2013.11.007. MR.3144659

[Ber65] S. J. Bernau, Unique representation of Archimedean lattice groups and normal Archimedean lattice rings, Proc. London Math. Soc. (3) 15 (1965), 599-631. MR0182661 (32 \#144)

[BH90a] Richard N. Ball and Anthony W. Hager, Epicomplete Archimedean l-groups and vector lattices, Trans. Amer. Math. Soc. 322 (1990), no. 2, 459-478, DOI 10.2307/2001709. MR943603 (91c:46009)

[BH90b] Richard N. Ball and Anthony W. Hager, Epicompletion of Archimedean l-groups and vector lattices with weak unit, J. Austral. Math. Soc. Ser. A 48 (1990), no. 1, 25-56. MR:1026835 (91g:06016)

[BH09] Karim Boulabiar and Anthony Hager, $\ell$-group homomorphisms between reduced Archimedean f-rings, Algebra Universalis 62 (2009), no. 4, 329-337, DOI 10.1007/s00012-010-0031-1. MR2670167 (2012b:06055)

[BHJK05] R. N. Ball, A. W. Hager, D. G. Johnson, and A. Kizanis, On the epicomplete monoreflection of an Archimedean lattice-ordered group, Algebra Universalis 54 (2005), no. 4, 417-434, DOI 10.1007/s00012-005-1952-y. MR2218854 (2007m:06021)

[BHJK09] R. N. Ball, A. W. Hager, D. G. Johnson, and A. Kizanis, A theorem and a question about epicomplete Archimedean lattice-ordered groups, Algebra Universalis 62 (2009), no. 2-3, 165-184, DOI 10.1007/s00012-010-0049-4. MR2661373 (2011j:06034)

[CM90] Paul Conrad and Jorge Martinez, Settling a number of questions about hyperArchimedean lattice-ordered groups, Proc. Amer. Math. Soc. 109 (1990), no. 2, 291296, DOI 10.2307/2047986. MR998733 (91i:06016)

[Con71] Paul Conrad, The essential closure of an Archimedean lattice-ordered group, Duke Math. J. 38 (1971), 151-160. MR0277457 (43 \#3190)

[Con74] Paul Conrad, The additive group of an $f$-ring, Canad. J. Math. 26 (1974), 1157-1168. MR0354487 (50 \#6965)

[GJ76] Leonard Gillman and Meyer Jerison, Rings of continuous functions, Springer-Verlag, New York, 1976. Reprint of the 1960 edition; Graduate Texts in Mathematics, No. 43. MR0407579 (53 \#11352)

[HJ61] M. Henriksen and D. G. Johnson, On the structure of a class of archimedean latticeordered algebras., Fund. Math. 50 (1961/1962), 73-94. MR0133698(24 \#A3524)

[HJ10] A. W. Hager and D. G. Johnson, Some comments and examples on generation of (hyper-)archimedean $\ell$-groups and $f$-rings (English, with English and French summaries), Ann. Fac. Sci. Toulouse Math. (6) 19 (2010), no. Fascicule Special, 75-100. MR2675722(2011i:06032)

[Hol03] W Charles Holland. The Black Swamp Problem Book. 2003.

[HR77] Anthony W. Hager and Lewis C. Robertson, Representing and ringifying a Riesz space, Symposia Mathematica, Vol. XXI (Convegno sulle Misure su Gruppi e su Spazi Vettoriali, Convegno sui Gruppi e Anelli Ordinati, INDAM, Rome, 1975), Academic Press, London, 1977, pp. 411-431. MR0482728 (58 \#2783)

[HS07] Horst Herrlich and George E. Strecker, Category theory, 3rd ed., Sigma Series in Pure Mathematics, vol. 1, Heldermann Verlag, Lemgo, 2007. An introduction. MR2377903 (2009c:18001)

[Jec03] Thomas Jech, Set theory, Springer Monographs in Mathematics, Springer-Verlag, Berlin, 2003. The third millennium edition, revised and expanded. MR.1940513 (2004g:03071) 
[Joh62] D. G. Johnson, On a representation theory for a class of Archimedean lattice-ordered rings, Proc. London Math. Soc. (3) 12 (1962), 207-225. MR0141685 (25 \#5082)

[Joh07] D. G. Johnson, A representation theorem revisited, Algebra Universalis 56 (2007), no. 3-4, 303-314, DOI 10.1007/s00012-007-2000-x. MR2318213 (2008d:06020)

Department of Mathematics and Computer Science, Wesleyan University, MiddleTOWn, Connecticut 06459

E-mail address: ahager@wesleyan.edu 